\title{
Intracranial Germinoma: Successful Management by Chemotherapy and Intensity Modulated Radiation Therapy
}

Issam Lalya ${ }^{1,2^{*}}$, Abdelhak Maghous ${ }^{1}$, El Amin Marnouche ${ }^{1}$, Noha Zaghba ${ }^{1}$, Khalid Andaloussi ${ }^{2}$, Mohamed Elmarjany ${ }^{3}$, Khalid Hadadi ${ }^{1}$, Hassan Sifat $^{1}$, Ismail Essadi $^{3}$ and Hamid Mansouri ${ }^{1}$

${ }^{1}$ Department of Radiotherapy, Mohamed V Military Teaching Hospital, Rabat, Morocco

${ }^{2}$ Faculty of Medicine and Pharmacy, Cadi Ayyad University, Marrakech, Morocco

${ }^{3}$ Department of Medical Oncology, Avicen Military Hospital, Marrakech, Morocco

“Corresponding author: Issam Lalya, Department of Radiotherapy, Mohamed V Military Teaching Hospital, Rabat, Morocco, Tel: 00212661572770; E-mail: issamlalya@yahoo.fr

Received date: October 04, 2017; Accepted date: October 17, 2017; Published date: October 25, 2017

Copyright: ( 2017 Lalya I, et al. This is an open-access article distributed under the terms of the Creative Commons Attribution License, which permits unrestricted use, distribution, and reproduction in any medium, provided the original author and source are credited.

\begin{abstract}
Introduction: Germinomas are malignant intracranial germ cell tumors, usually found in pineal region. These tumors are exquisitely sensitive to radiation. Whole ventricle radiation (RT) with a boost field to the primary tumor is the standard treatment for localized germinomas. RapidArc radiotherapy is considered an excellent technological advance that shows great potential for producing highly conformal doses to treatment volumes.
\end{abstract}

Case report: We present a case of intracranial germinoma who achieved a partial remission after four courses of chemotherapy induction. The patient received whole ventricular radiation therapy followed by a boost to the tumor and its margin to a total dose of $40 \mathrm{~Gy}$ using RapidArc radiotherapy over the course of 5 weeks. Any toxicity has been noted during the treatment. Early post treatment imaging showed a complete response.

Conclusion: This case demonstrates a successful management of localized intracranial germinoma by RapidArc radiotherapy that allowed a highly conformal volumetric dose distribution with optimal sparing of surrounding areas of the brain.

Keywords: Intracranial germinomas; Chemotherapy; Radiotherapy; RapidArc

\section{Introduction}

Germinomas, the most frequent intracranial germ cell tumors (GCTs), are rare tumors of children and young adults. They represent about $0.5 \%$ to $3 \%$ of all primary central nervous system tumors in North America and Europe [1]. These tumors arise almost exclusively from midline structures. The two most frequent sites are the pineal gland and the suprasellar regions [2], but they can also arise in the basal ganglia, thalamus, cerebral hemisphere, and cerebellum [3]. Tumor seeding or multiple tumor nodules along the lateral and third ventricles are observed in about $10 \%$ of patients. Intracranial germinomas are highly radiosensitive. Most contemporary series have reported long-term progression free survival (PFS) rates $>90 \%$ for patients with localized, pure germinomas after radiation therapy (RT) with or without chemotherapy [4-8]. Therefore, RT is the standard treatment for localized intracranial germinomas. We report an excellent complete remission case of pineal gland germinoma who achieved a partial remission after neoadjuvant chemotherapy, successfully managed by RapidArc radiotherapy allowing a highly conformal volumetric dose distribution.

\section{Case Report}

A 25-year-old Arabic male without past medical history presented with a 6-month history of headaches and vomiting. Neurological examination revealed no abnormalities. Magnetic Resonance Imaging
(MRI) of the brain revealed a mass in the pineal region with obstructive hydrocephalus (Figure 1). It was a voluminous polycyclic intra axial mesencephalic-diencephalic masse (measuring $77 \times 44 \mathrm{~mm}$ in diameter) centered within the pineal gland and the two thalamic nuclei with cystic components showing heterogeneous enhancement with gadolinium suggestive of a pineal germinoma. The patient underwent a ventriculoperitoneal shunt operation with tumor biopsy. A subsequent clinical evolution was marked by the disappearance of symptoms. Histological analysis was compatible with a germinoma. We didn't find similar lesions in testicles or elsewhere in the body.

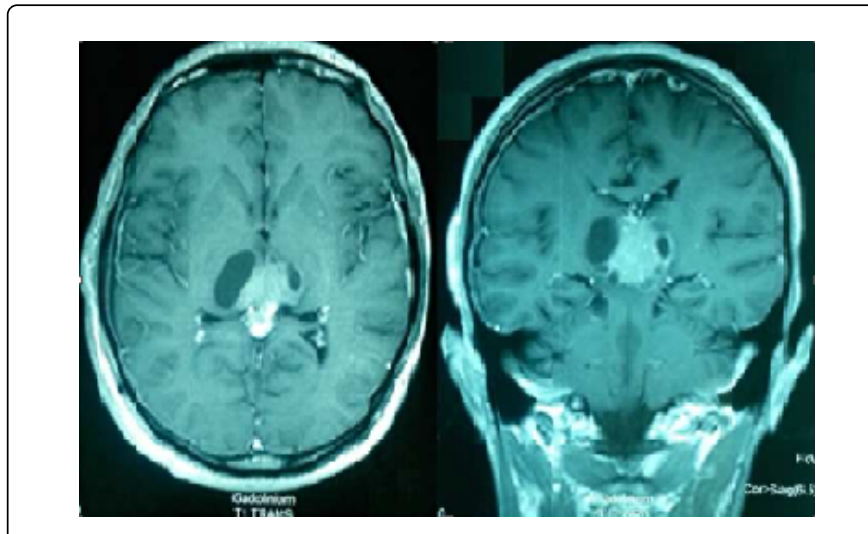

Figure 1: Axial and coronal T1-weighted contrast-enhanced MRI showing a heterogeneously enhancing mass in the pineal region. 
Citation: Lalya I, Maghous A, Marnouche EA, Zaghba N, Andaloussi K, et al. (2017) Intracranial Germinoma: Successful Management by Chemotherapy and Intensity Modulated Radiation Therapy. J Nucl Med Radiat Ther 8: 341. doi:10.4172/2155-9619.1000341

Page 2 of 3

The patient was treated primary with neoadjuvant chemotherapy based of bleomycin, etoposide and cisplatin (BEP). After four courses of BEP chemotherapy, partial remission of more than $50 \%$ was noted on comparative MRI of the brain. Thereafter, the patient received whole ventricular radiation therapy of $24 \mathrm{~Gy}$ in 15 daily fractions of 1.6 Gy given in 3 weeks followed by a boost to the gross tumor volume with margin of $16 \mathrm{~Gy}$ in 10 daily fractions of $1.6 \mathrm{~Gy}$ given in 2 weeks to give a total dose of 40 Gy in 25 fractions of 1.6 Gy given in 5 weeks using dual arc and two plans RapidArc radiotherapy allowing a highly conformal volumetric dose distribution (Figure 2). Early post treatment imaging demonstrated complete remission of the tumor (Figure 3).
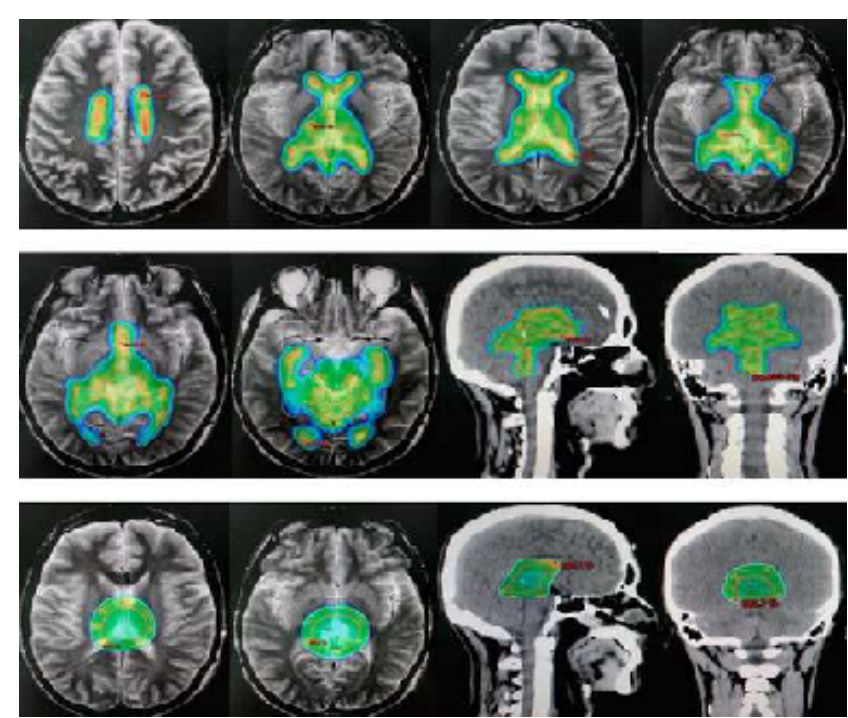

Figure 2: Sequential images of MRI and computed tomography scan dosimetry showing a conformal volumetric dose distribution by RapidArc radiotherapy.

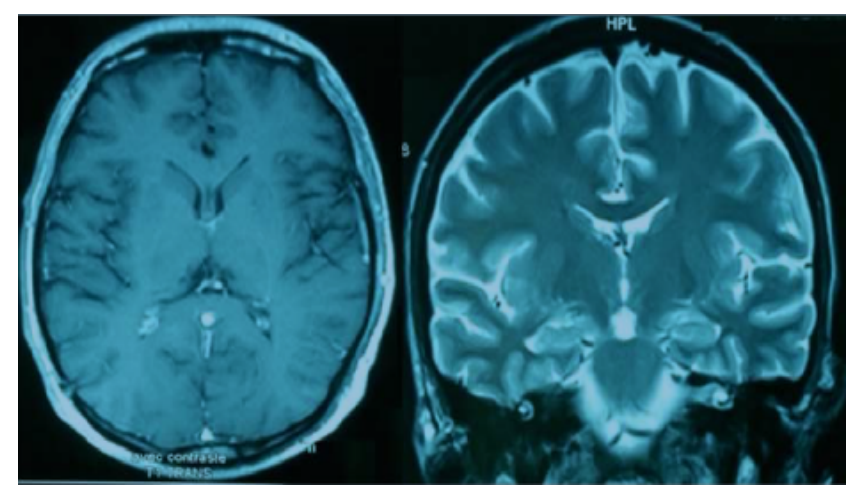

Figure 3: Axial T1-weighted contrast-enhanced and coronal T2weighted early post treatment MRI showing a complete remission of the tumor.

\section{Discussion}

Intracranial germinomas are highly sensitive to radiation. Therefore, whole ventricular RT is the current standard treatment for localized germinomas. RapidArc radiotherapy is an advanced radiation technique that allows a highly conformal volumetric dose distribution to target volumes without result in unwanted radiation to normal areas of brain and compromising control of the tumor $[9,10]$. Complete remission of our case support the superiority of RapidArc system to manage localized intracranial germinomas.

In the past, patients with localized intracranial germinomas received 36 Gy craniospinal irradiation (CSI) and a boost to the tumor bed for a total of 50 to $54 \mathrm{~Gy}$. Subsequent studies showed that replacing CSI with whole-ventricle irradiation in patients with localized intracranial germinomas resulted in a low spinal failure rate [11-15]. The patterns of relapse following whole-ventricle RT compared to CSI were not significantly different; suggesting that recurrent intracranial germinomas after RT are unlikely to be related to a volume reduction in RT. Whole-ventricle RT with an additional boost to the tumor therefore replaced CSI in the treatment of localized germinoma. However, CSI is reserved only for patients with disseminated disease or tumors who do not demonstrate either a complete response or partial response to chemotherapy.

The importance of whole-ventricle RT was showed in a series of 35 patients with localized intracranial germinomas who did not receive CSI [15]. In this cohort, 21 patients were treated with whole-ventricle irradiation, none of whom developed recurrent disease. In contrast, 5 of $14(36 \%)$ who received only focal tumor irradiation had recurrent tumors within the ventricular system but outside the primary treatment field. Similarly, in a second series, the recurrence rate for patients receiving localized irradiation without ventricular coverage was higher than in those receiving CSI (28 vs. 2\%) [4].

Based upon the excellent response and survival outcome of children and adults with extra cranial GCTs, neoadjuvant chemotherapy was given to patients with localized intracranial germinomas, followed by a reduced dose and volume of $\mathrm{RT}$, in an effort to minimize toxicity. Clinical trials have indicated that cure rates of nearly $100 \%$ can be achieved in germinomas with chemotherapy and radiotherapy and suggested that neoadjuvant chemotherapy allowed the reduction of both the dose and volume of RT without compromising PFS [16-21]. Although the majority of germinomas are sensitive to chemotherapy, a chemotherapy alone approach has been associated with an unacceptable rate of relapse.

\section{Conclusion}

Radiation therapy is the cornerstone of treatment for localized intracranial germinoma. Currently, the standard of care for radiation dose in localized germinoma is 21 to 24 Gy to the whole ventricle and an additional boost to the tumor bed for a total dose of 40 to $45 \mathrm{~Gy}$. Despite the promise of neoadjuvant chemotherapy, further reduction of the total RT dose should only be done within the context of a prospective randomized trial. The best way to optimize treatment is the use of innovative techniques of radiation therapy such RapidArc allowing optimal coverage of target volumes and dose escalation, with minimal toxicity.

\section{Consent}

Written informed consent was obtained from the patient for publication of this Case Report and any accompanying images. A copy of the written consent is available for review by the Editor-in-Chief of this journal. 
Citation: Lalya I, Maghous A, Marnouche EA, Zaghba N, Andaloussi K, et al. (2017) Intracranial Germinoma: Successful Management by Chemotherapy and Intensity Modulated Radiation Therapy. J Nucl Med Radiat Ther 8: 341. doi:10.4172/2155-9619.1000341

Page 3 of 3

\section{Author's Contribution}

Issam Lalya, Abdelhak Maghous El Amin Marnouche, Ismail Essadi and Hamid Mansouri were the major contributors in writing the manuscript. Noha Zaghba, Khalid Andaloussi, Mohamed Elmarjany, Khalid Hadadi and Hassan Sifat cared for the patient during her time in the hospital and assisted in data collection and the preparation of the manuscript. All authors read and approved the final manuscript.

\section{Acknowledgement}

The authors would like to thank the team of radiation therapists for performing daily image control before the treatment, and the social department of Moroccan Royal Armed Force for patient's psycho social support.

\section{Competing Interests}

The authors declare that they have no competing interests.

\section{Data Availability}

Data sharing not applicable to this article as no datasets were generated or analysed during the current study.

\section{Funding}

Special thanks are due to the Faculties of Medicine and Pharmacy of Rabat and Marrakesh; the source(s) of funding for all authors.

\section{References}

1. Echevarría ME, Fangusaro J, Goldman S (2008) Pediatric central nervous system germ cell tumors: A review. Oncologist 13: 690.

2. Liang L, Korogi Y, Sugahara T, Shigematsu Y, Ikushima I, et al. (2002) MRI of intracranial germ-cell tumours. Neuroradiology 44: 382-388.

3. Tomura N, Takahashi S, Kato K (2000) Germ cell tumors of the central nervous system originating from non-pineal regions: CT and MR features. Comput Med Imaging Graph 24: 269-276.

4. Bamberg M, Kortmann RD, Calaminus G, Becker G, Gobel U, et al (1999) Radiation therapy for intracranial germinoma: Results of the German cooperative prospective trials MAKEI 83/86/89. J Clin Oncol 17: 2585-2592.

5. Haddock MG, Schild SE, Scheithauer BW, Schomberg PJ (1997) Radiation therapy for histologically confirmed primary central nervous system germinoma. Int J Radiat Oncol Biol Phys 38: 915.

6. Hardenbergh PH, Golden J, Billet A, Tarbell NJ, Silver B, et al. (1997) Intracranial germinoma: The case for lower dose radiation therapy. Int $\mathrm{J}$ Radiat Oncol Biol Phys 39: 419.

7. Wolden SL, Wara WM, Larson DA, Prados MD, Sneed PK, et al. (1995) Radiation therapy for primary intracranial germ-cell tumors. Int J Radiat Oncol Biol Phys 32: 943-949.
8. Jensen AW, Laack NN, Buckner JC, Paula S, Brown PD, et al. (2010) Long-term follow-up of dose-adapted and reduced-field radiotherapy with or without chemotherapy for central nervous system germinoma. Int J Radiat Oncol Biol Phys 77: 1449-1456.

9. Katsuyuki S, Takashi M, Masahiro H (2010) Dosimetric advantage of intensity-modulated radiotherapy for whole ventricles in the treatment of localized intracranial germinoma. Int J Radiat Oncol Biol Phys 82: e273-280.

10. Sharon QX, Stinauer M, Rogers B, Madden JR, Wilkening GN, et al. (2012) Potential for improved intelligence quotient using volumetric modulated arc therapy compared with conventional 3-dimensional conformal radiation for whole-ventricular radiation in children. Int J Radiat Oncol Biol Phys 84: 1206-1211.

11. Dattoli MJ, Newall J (1990) Radiation therapy for intracranial germinoma: The case for limited volume treatment. Int J Radiat Oncol Biol Phys 19: 429-433.

12. Linstadt D, Wara WM, Edwards MS, Sheline GE, Hudgins RJ (1988) Radiotherapy of primary intracranial germinomas: the case against routine craniospinal irradiation. Int J Radiat Oncol Biol Phys 15: 291.

13. Rogers SJ, Mosleh-Shirazi MA, Saran FH (2005) Radiotherapy of localised intracranial germinoma: Time to sever historical ties? Lancet Oncol 6: 509-519.

14. Shikama N, Ogawa K, Tanaka S, Toita T, Uno T, et al. (2005) Lack of benefit of spinal irradiation in the primary treatment of intracranial germinoma: A multiinstitutional, retrospective review of 180 patients. Cancer 104: 126-134.

15. Haas-Kogan DA, Missett BT, Wara WM, Prados MD, Fisch BM, et al. (2003) Radiation therapy for intracranial germ cell tumors. Int J Radiat Oncol Biol Phys 56: 511-518.

16. Buckner JC, Peethambaram PP, Smithson WA, Groover RV, RAffel C, et al. (1999) Phase II trial of primary chemotherapy followed by reduceddose radiation for CNS germ cell tumors. J Clin Oncol 17: 933-940.

17. Fouladi M, Grant R, Baruchel S, Malkin D, Greenberg M, et al. (1998) Comparison of survival outcomes in patients with intracranial germinomas treated with radiation alone versus reduced-dose radiation and chemotherapy. Childs Nerv Syst 14: 596-601.

18. Douglas JG, Rockhill JK, Olson JM, Russell J, Richard G, et al. (2006) Cisplatin-based chemotherapy followed by focal, reduced-dose irradiation for pediatric primary central nervous system germinomas. J Pediatr Hematol Oncol 28: 36-39.

19. Kretschmar C, Kleinberg L, Greenberg M, Burger P, Holmes E, et al. (2007) Pre-radiation chemotherapy with response-based radiation therapy in children with central nervous system germ cell tumors: a report from the Children's Oncology Group. Pediatr Blood Cancer 48: 285-291.

20. Khatua S, Dhall G, O'Neil S (2010) Treatment of primary CNS germinomatous germ cell tumors with chemotherapy prior to reduced dose whole ventricular and local boost irradiation. Pediatr Blood Cancer 55: 42

21. Kellie SJ, Boyce H, Dunkel IJ, Finlay JL, Diez B, et al. (2004) Intensive cisplatin and cyclophosphamide-based chemotherapy without radiotherapy for intracranial germinomas: Failure of a primary chemotherapy approach. Pediatr Blood Cancer 43: 126-133. 